\title{
High biologically effective dose radiation therapy using brachytherapy in combination with external beam radiotherapy for high-risk prostate cancer
}

\author{
Keisei Okamoto, MD, PhD', Akinori Wada, MD², Naoaki Kohno, MD, PhD³ \\ 'Department of Brachytherapy for Prostate Cancer, ${ }^{2}$ Department of Urology, ${ }^{3}$ Department of Radiology. Shiga University of Medical Science, \\ Shiga, Japan
}

\begin{abstract}
Purpose: To evaluate the outcomes of high-risk prostate cancer patients treated with biologically effective dose (BED) $\geq 220$ Gy of high-dose radiotherapy, using low-dose-rate (LDR) brachytherapy in combination with external beam radiotherapy (EBRT) and short-term androgen deprivation therapy (ADT).

Material and methods: From 2005 to 2013, a total of 143 patients with high-risk prostate cancer were treated by radiotherapy of BED $\geq 220$ Gy with a combination of LDR brachytherapy, EBRT, and androgen deprivation therapy (ADT). The high-risk patients in the present study included both high-risk and very high-risk prostate cancer. The number of high-risk features were: 60 patients with 1 high-risk factor (42\%), 61 patients with 2 high-risk factors $(43 \%)$, and 22 patients with 3 high-risk factors (15\%) including five N1 disease. External beam radiotherapy fields included prostate and seminal vesicles only or whole pelvis depending on the extension of the disease. Biochemical failure was defined by the Phoenix definition.

Results: Six patients developed biochemical failure, thus providing a 5-year actual biochemical failure-free survival (BFFS) rate of $95.2 \%$. Biochemical failure was observed exclusively in cases with distant metastasis in the present study. All six patients with biochemical relapse had clinical failure due to bone metastasis, thus yielding a 5-year freedom from clinical failure (FFCF) rate of $93.0 \%$. None of the cases with N1 disease experienced biochemical failure. We observed four deaths, including one death from prostate cancer, therefore yielding a cause-specific survival (CSS) rate of $97.2 \%$, and an overall survival (OS) rate of $95.5 \%$

Conclusions: High-dose (BED $\geq 220 \mathrm{~Gy}$ ) radiotherapy by LDR in combination with EBRT has shown an excellent outcome on BFFS in high-risk and very high-risk cancer, although causal relationship between BED and BFFS remain to be explained further.

Key words: LDR brachytherapy, high-risk, prostate cancer, seeds.

\section{Purpose}

High-risk prostate cancer is a category of prostate cancer that includes an aggressive tumor and/or a high tumor burden [1,2]. Importantly, inadequate local control in the management of the high-risk prostate cancer patients leads to metastasis or death. Therefore, for optimal radiotherapy results in high-risk prostate cancer, a good local control by dose escalation is crucial $[1,3]$.

The use of prostate brachytherapy provides the advantage of safe delivery of a high biologically effective dose (BED) to the prostate $[1,2,3,4]$. The advantage of combination therapy with low-dose-rate (LDR) brachytherapy and external beam radiotherapy (EBRT) has been recently confirmed by the ASCENDE-RT (Androgen Suppression

Combined with Elective Nodal and Dose Escalated Radiation Therapy) randomized trial [5]. The combination of LDR and EBRT is associated with prostate cancer specific mortality in some prostate cancer patients [6]. In terms of radiation dose, Stone et al. have shown that patients with Gleason 8-10 disease receiving a biologically effective dose $(B E D) \geq 220$ Gy by combination therapy with LDR brachytherapy and EBRT, obtained improvement in biochemical failure-free survival (BFFS) [7]. In order to study the efficacy and toxicity of the combination therapy of BED $\geq 220$ Gy by tri-modality (LDR brachytherapy in combination with EBRT and short term androgen deprivation therapy [ADT]) in a Japanese population, we analyzed the clinical outcome of 143 high-risk and very high-risk patients, including those with N1 disease.
\end{abstract}




\section{Material and methods}

\section{Patients}

This retrospective and observational research has been conducted in accordance with the Helsinki Declaration. This study has been approved and monitored by our institutional ethics committee (Shiga University of Medical Science: 23-133 and 23-196). For individual data usage including images, we have obtained separate informed consent from patients. From 2005 to 2013, a total of 143 patients with high-risk prostate cancer were treated by a combination of LDR brachytherapy, external beam radiotherapy, and ADT. These patients had a minimum follow-up time of two years. The high-risk patients in the present study included both high-risk and very high-risk prostate cancer as classified in the current National Cancer Network Criteria (http:/ / www.nccn.org). Briefly, patients were defined as high-risk if they fulfilled at least one of the following criteria: prostate-specific antigen

Table 1. Patient characteristics

\begin{tabular}{|c|c|}
\hline Variable & $n=143(\%)$ \\
\hline \multicolumn{2}{|l|}{ Age, years } \\
\hline Median (range) & $66.9(55-82)$ \\
\hline \multicolumn{2}{|l|}{ PSA at diagnosis, $\mathrm{ng} / \mathrm{ml}$} \\
\hline$<10$ & $41(29 \%)$ \\
\hline $10-20$ & $43(30 \%)$ \\
\hline$>20$ & $59(41 \%)$ \\
\hline Median (range) & $20.76(4-130)$ \\
\hline \multicolumn{2}{|l|}{ Gleason score } \\
\hline 6 & $6(4 \%)$ \\
\hline 7 & $41(29 \%)$ \\
\hline 8 & $68(48 \%)$ \\
\hline 9 & $25(17 \%)$ \\
\hline 10 & $3(2 \%)$ \\
\hline \multicolumn{2}{|l|}{ Tumor stage } \\
\hline T1c & $13(9 \%)$ \\
\hline $\mathrm{T} 2 \mathrm{a}$ & $4(3 \%)$ \\
\hline $\mathrm{T} 2 \mathrm{~b}$ & $24(17 \%)$ \\
\hline $\mathrm{T} 2 \mathrm{C}$ & $11(8 \%)$ \\
\hline T3a & $70(48 \%)$ \\
\hline $\mathrm{T} 3 \mathrm{~b}$ & $20(14 \%)$ \\
\hline $\mathrm{T} 4$ & $1(1 \%)$ \\
\hline \multicolumn{2}{|l|}{ Metastasis } \\
\hline No metastasis & $138(97 \%)$ \\
\hline Regional lymph node metastasis & $5(3 \%)$ \\
\hline
\end{tabular}

PSA - prostate specific antigen
(PSA) level higher than $20 \mathrm{ng} / \mathrm{ml}$, and/or Gleason score $>8$, and/or clinical stage T3. We also enrolled T3b-T4 disease in this study. Gleason scores of all biopsy specimens were reviewed by our central pathologist before the treatment. Clinical $\mathrm{T}$ stage was determined by a combination of magnetic resonance imaging (MRI) and digital examination. All patients had bone scans and computed tomography (CT) of the pelvis to check the presence of bone metastasis and lymph node metastasis.

In addition, we enrolled regional N1 disease patients in this study if the nodal involvement of the pelvis was limited (one or two nodal metastases). Lymph node metastasis was finally confirmed on CT by shrinkage of lymph nodes through neoadjuvant ADT. Clinical characteristics of the patients (PSA, Gleason score clinical T stage) in the present study is shown in Table 1 . The distribution of the number of high-risk factors in the present study is shown in Table 2 .

\section{Treatment}

All patients were treated by combination therapy with LDR brachytherapy, EBRT, and ADT. Androgen deprivation therapy included neoadjuvant (six months) and adjuvant (six months) settings across the seed implantation. Androgen deprivation therapy consisted of gonadotropin-releasing hormone agonist injection and anti-androgen. Low-dose-rate brachytherapy implantation in the prostate was conducted with ${ }^{125}$ I seeds using real-time ultrasound guided technique [8]. Radioactive seeds were deposited into the prostate using a Mick applicator (Mick Radio-Nuclear Instruments, Inc., Mount Vernon, NY, USA). Seminal vesicle implantation was added based on the advancement of the disease such as seminal vesicle involvement or tumor location adjacent to the seminal vesicle [9]. The prescription dose of seed implantation was set at $110 \mathrm{~Gy}$ [3].

To achieve a BED of $220 \mathrm{~Gy}, \mathrm{D}_{90}$ of $130 \mathrm{~Gy}$ have to be delivered (post-implant $\mathrm{D}_{90}$ ) by using ${ }^{125}$ I seed implantation in combination with the $45 \mathrm{~Gy}$ of EBRT in $1.8 \mathrm{~Gy}$ fraction [3]. In order to secure $130 \mathrm{~Gy}$ of $\mathrm{D}_{90}$ at post ${ }^{125} \mathrm{I}$ seed implantation, we usually set $\mathrm{D}_{90}$ at implantation from 135 Gy to $145 \mathrm{~Gy}$.

By complying with the above-mentioned implantation policy, the clinical target volume (CTV) was covered with $130 \mathrm{~Gy}$, and the CTV with margin was covered with 110 Gy (prescription dose).

Post-implant dosimetry with CT and MRI guidance was carried out at one month after seed implantation. Supplemental EBRT was delivered four to eight weeks after seed implantation. External beam radiotherapy consisted of a median dose of $45 \mathrm{~Gy}$, given in $1.8 \mathrm{~Gy}$ fractions via a three-dimensional conformal technique. Clinical target volume was designed as the entire prostate and

Table 2. Number of high-risk factors

\begin{tabular}{ll}
1 & $60(42 \%)$ \\
\hline 2 & $61(43 \%)$ \\
\hline 3 & $22(15 \%)$
\end{tabular}


seminal vesicle. Planning target volume (PTV) included CTV-block with a $15 \mathrm{~mm}$ margin, except at the prostatorectal interface where 7-10 $\mathrm{mm}$ margin was used.

For each case, the BED was calculated from the prostate $\mathrm{D}_{90}$ and EBRT dose using the formula described previously [4]: the EBRT dose was determined, so that the total BED would be higher than $220 \mathrm{~Gy}$ as long as $\mathrm{UD}_{30}$ and $R_{100}$ were tolerable. Upon supplemental EBRT, whole pelvis external beam radiotherapy (45 Gy) was applied in $\mathrm{N} 1$ cases or in some of the very high-risk patients, although the usual EBRT fields included prostate and seminal vesicles only with margin.

\section{Toxicity}

Acute toxicity was defined if symptoms developed within the first year after seed implantation. Late toxicity was defined if any kind of symptom developed after one year, or if a symptom occurred within the first year and persisted for more than one year. Toxicity was recorded by the Common Terminology Criteria for Adverse Events version 4.0.

\section{Follow-up and statistical analysis}

Scheduled follow-up was done by PSA blood test and physical examination every three months for the first two years, followed by every six months thereafter. Duration of follow-up was calculated from the end of the supplemental EBRT. Patients had a minimum follow-up time of two years (median 52 months; range, 28-131 months). Actuarial survival curves were calculated by the Kaplan Meier method to determine biochemical relapse-free survival (BFFS), freedom from clinical failure (FFCF) survival, cause-specific survival (CSS), and overall survival (OS). Biochemical failure was defined according to the Phoenix Definition [9]. The criterion for biochemical failure with subsequent PSA decrease to $<0.5 \mathrm{ng} / \mathrm{ml}$ without intervention was categorized as a benign bounce and was excluded from the biochemical failure group. Upon a true biochemical failure, we performed CT, MRI, bone scan, and rectal digital examination to evaluate whether biochemical failure was caused by distant metastasis or local failure. Biochemical relapse-free survival was calculated for all living patients and reflected biochemical failures. Freedom from clinical failure survival rate was calculated for all living patients and presented clinical failure events (local, regional, and distant failure). Cause-specific survival reflected prostate cancer-specific death. Overall survival presented all deaths, cancer related or unrelated.

\section{Results}

\section{Dosimetric parameters and biologically effective dose}

Dosimetric parameters of seed implantation at one month and total BED of 143 high-risk patients are shown in Table 3. Total BED was over 220 Gy in 106 cases $(74 \%)$ over 215 Gy in 119 cases (83\%), over 210 Gy in 128 cases $(90 \%)$, over 205 Gy in 131 cases (92\%), and over 200 Gy in 136 cases $(95 \%)$ (Table 4$)$.

\section{Efficacy of the treatment}

Of the 143 high-risk patients, six developed PSA failure, yielding an actuarial BFFS rate of $95.2 \%$ at 5 years (Figure 1). These six patients with BFFS had a clinical failure due to bone metastasis, yielding a FFCF rate of $93.0 \%$ at 5 years (Figure 1). According to MRI and rectal digital examination, all six patients with biochemical failure and clinical failure had no evidence of local failure. One of these patients with biochemical failure and clinical failure died of prostate cancer, yielding a CSS rate of $97.2 \%$ at 5 years (Figure 1). Another three patients died during follow-up, including two patients who died of cerebral infarction, and one patient who died of myelodysplastic syndrome, yielding an OS rate of $95.5 \%$ at 5 years (Figure 1 ).

\section{Outcome of regional nodal metastasis}

Five cases with regional nodal metastasis treated by tri-modality with whole pelvis EBRT showed neither biochemical failure nor clinical failure. A representative case with regional nodal metastasis treated in the present study is shown in Figure 2.

\section{Toxicity}

Acute grade 2 gastrointestinal (GI) and genitourinary (GU) toxicity was experienced by two patients $(1.3 \%)$ and 15 patients $(10.4 \%)$, respectively. Late grade 2 gastroin-

Table 3. Dosimetric parameters of seeds implantation and total biologically effective dose (BED)

\begin{tabular}{lc} 
Variables & Median (range) \\
\hline Prostate $D_{90}$ (Gy) & $133.1(95.7-153.9)$ \\
\hline $\mathrm{V}_{100}(\%)$ & $97.3(82.1-99.8)$ \\
\hline $\mathrm{UD}_{30}$ (Gy) & $166.5(126.2-231.9)$ \\
\hline $\mathrm{R}_{100}$ (cc) & $0.29(0-2.0)$ \\
\hline Total BED (Gy) & $220.9(185.5-236.4)$
\end{tabular}

BED - biologically effective dose, $D_{90}$ - minimal dose (Gy) received by $90 \%$ of the prostate, $V_{100}$ - the percentage prostate volume receiving $100 \%$ of the prescribed minimal peripheral dose, $U D_{30}-$ minimal dose (Gy) by $30 \%$ of the ure thra, $R_{100}$ - rectal volume $(\mathrm{ml})$ receiving $100 \%$ of the prescribed dose

Table 4. Distribution of total biologically effective dose (BED)

\begin{tabular}{lc} 
Variables & Number of cases \\
\hline $190>B E D \geq 185$ & 1 \\
\hline $195>B E D \geq 190$ & 1 \\
\hline $200>B E D \geq 195$ & 5 \\
\hline $205>B E D \geq 200$ & 5 \\
\hline $210>B E D \geq 205$ & 3 \\
\hline $215>B E D \geq 210$ & 9 \\
\hline $220>B E D \geq 215$ & 13 \\
\hline$B E D \geq 220$ & 106
\end{tabular}


A

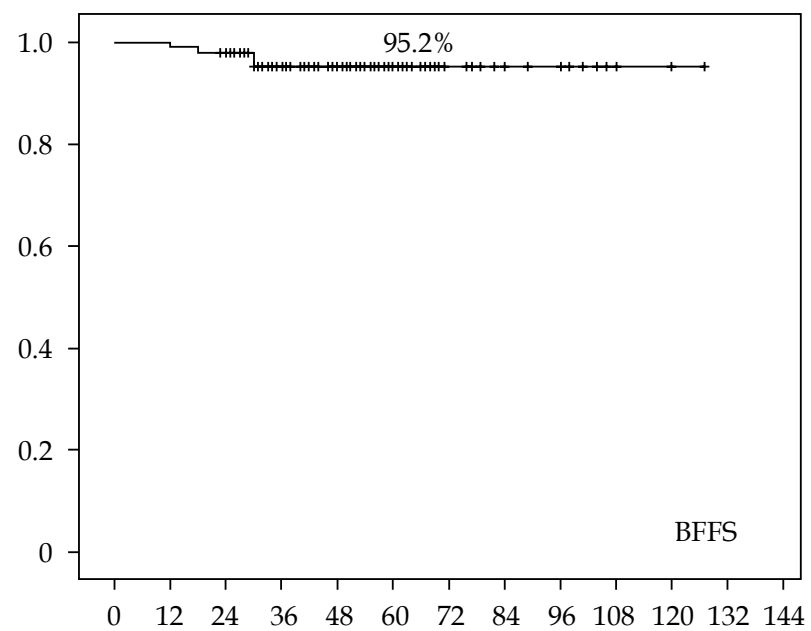

C

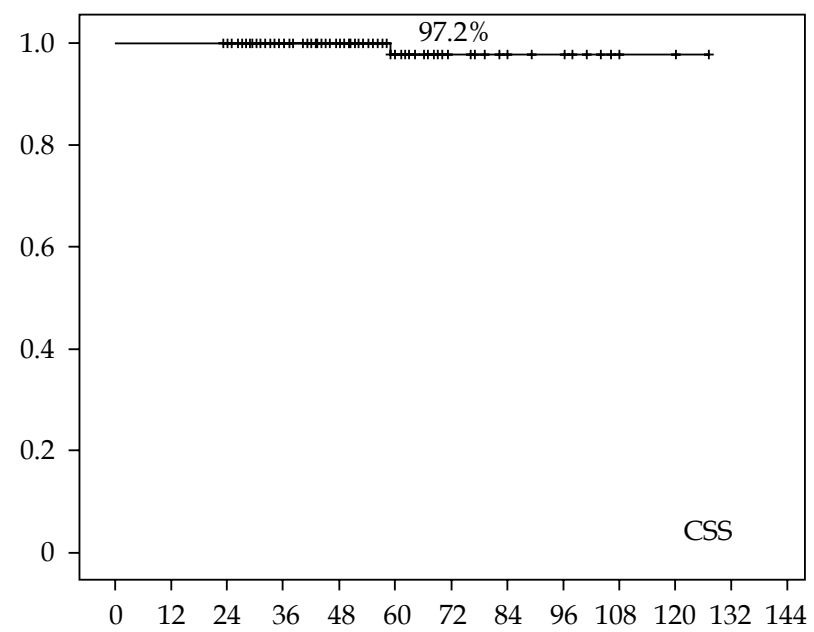

B

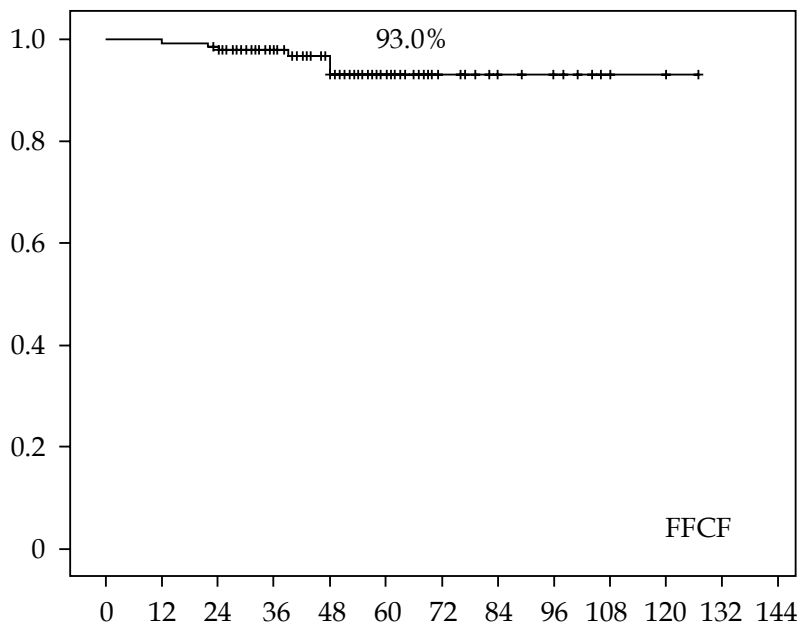

D

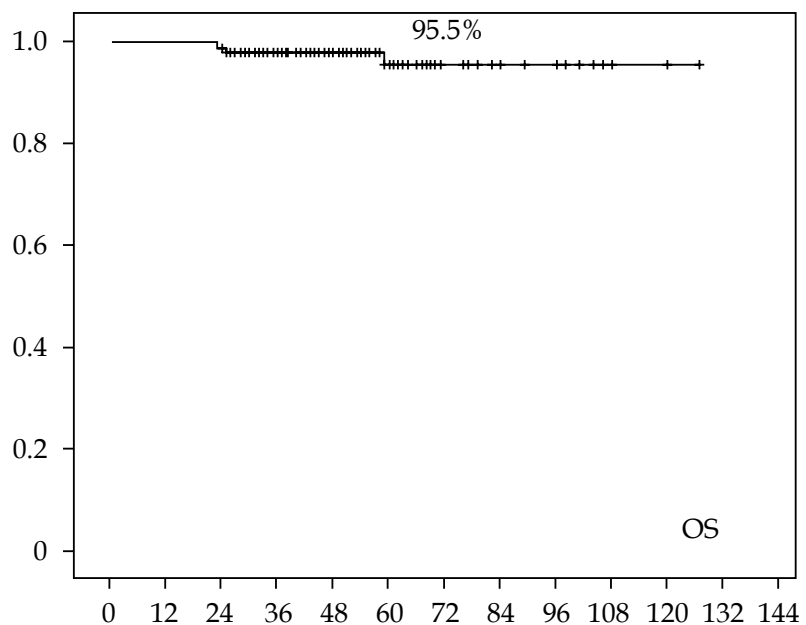

Fig. 1. Kaplan-Meier biochemical failure-free survival (BFFS), freedom from clinical failure (FFCF), cause-specific survival (CSS), and overall survival (OS). $\mathrm{Y}$ axis: survival probability; $\mathrm{X}$ axis: follow-up time after completion of external beam radiotherapy (EBRT) (months)

testinal (GI) and genitourinary (GU) toxicity was experienced by three patients $(2.0 \%)$ and six patients $(4.1 \%)$, respectively. One patient received preventive hyperbaric oxygen therapy for grade 2 rectal bleeding due to pancytopenia caused to end-stage renal malfunction. One patient received platelet transfusion for idiopathic platelet deficiency, although the patient did not have rectal bleeding. None of the patients experienced grade $>3$ acute or late toxicity. None of the patients experienced urethral stricture, transurethral resection of prostate (TURP), or recto-urethral fistula.

\section{Pattern of biochemical failure and number of high-risk features}

Six out of the six patients $(100 \%)$ with biochemical failure developed distant metastasis due to bone metastasis. Of those, 4 patients experienced one high-risk feature $(6.7 \%$ of the cases with one risk), one patient had two high-risk features (1.6\% of the cases with two risks), and one patient had three high-risk features $(4.5 \%$ of the cases with three risks). Thus, the number of high-risk features did not have impact on disease recurrence. All the six patients showed a similar pattern of biochemical failure. A continuous PSA increase was observed after cessation of ADT. The median (range) time to biochemical failure was 23 (12-30) months with $100 \%$ failing within the first 3 years. The median (range) time from biochemical failure to distant metastasis was 9 (0-18) months.

\section{Discussion}

The present data have shown an excellent clinical outcome by high-dose (BED > $220 \mathrm{~Gy}$ ) radiotherapy using LDR in combination with EBRT and ADT. The BED in the present study is the highest compared with those in the previous studies using LDR brachytherapy $[10,11]$.

To achieve a BED of $220 \mathrm{~Gy}, \mathrm{D}_{90}$ of $130 \mathrm{~Gy}$ had to be delivered (post-implant $\mathrm{D}_{90}$ ) by using ${ }^{125}$ I seed implantation in combination with the 45 Gy of EBRT in $1.8 \mathrm{~Gy}$ fraction. Several groups have demonstrated effectiveness of LDR in combination with EBRT and ADT for high- 

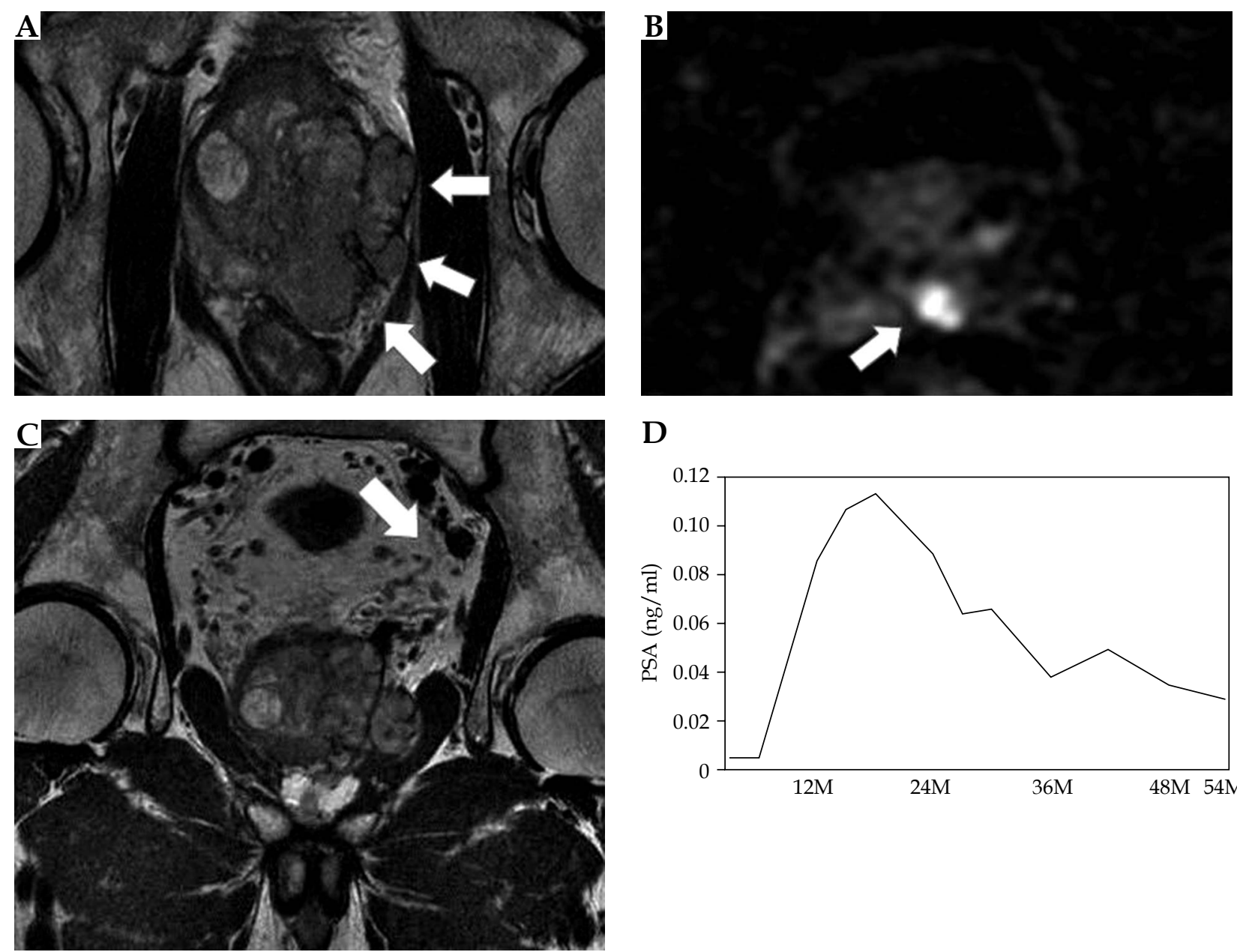

D

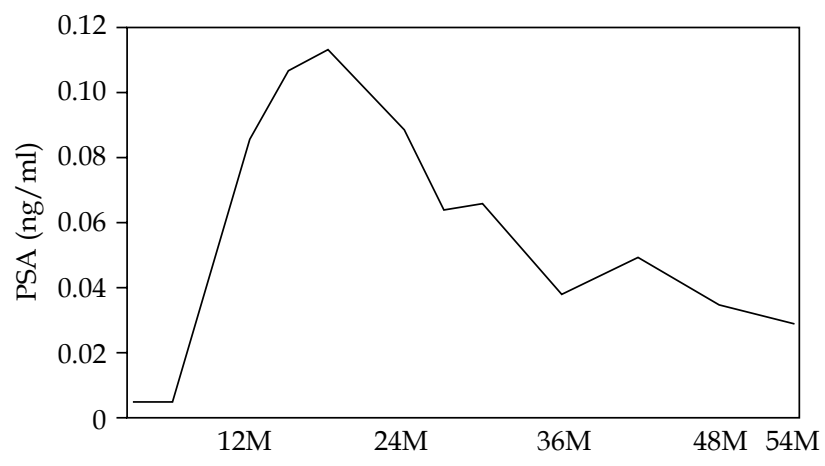

Fig. 2. Representative T3bN1 case treated by tri-modality with whole pelvis external beam radiotherapy (EBRT). Case: 68-yearold man with initial prostate-specific antigen (PSA) $65 \mathrm{ng} / \mathrm{ml}$. The needle biopsy Gleason's score was 4+4. Clinical stage was T3bN1. Magnetic resonance imaging shows (A) a bulky prostate tumor extending over the capsule and compressing the rectal wall (B) with seminal vesicle invasion, and (C) nodal metastasis as indicated by white arrows. The patient was treated by combination therapy with low-dose-rate (LDR) brachytherapy, whole pelvis EBRT, and androgen deprivation therapy (ADT). Upon seeds implantation, they were implanted in seminal vesicle as well as prostate [15]; (D) PSA change after treatment: PSA shows temporal increase after cessation of ADT, but continuous decrease was observed thereafter. Y axis shows the period (months) from completion of the EBRT

risk prostate cancer $[10,11]$. They have shown favorable biochemical relapse-free rates at 5 years of $79 \%[10]$ and $84.8 \%$ [11]. In terms of profiles of high-risk prostate cancer patients, it should be noted that this study consists of a significant number of very high-risk prostate cancer patients with two high-risk factors (43\%) and three highrisk factors $(15 \%)$, including 5 cases $(3 \%)$ with N1 disease and 21 cases $(15 \%)$ with T3b or T4 disease. Furthermore, the initial PSA was higher than that of the previous studies using LDR in combination with EBRT for high-risk prostate cancer [10,11].

The present data have shown a 5 year BFFS at $95.2 \%$. Similarly, high-dose-rate (HDR) brachytherapy is a modality that can deliver high BED in combination with EBRT. A previous study using HDR-based radiotherapy in combination with EBRT and long-term ADT, demonstrated a favorable 5 year BFFS of $85.1 \%$ with a median follow-up of 44 months for high-risk and very high-risk prostate cancer patients, including some in the intermediate risk category [12]. The data showed that biochemical failure occurred at a median of 40 months [12].

Kamrava et al. also reported on HDR based radiotherapy in combination with EBRT; they observed biochemical relapse in $14 \%$ of high-risk prostate cancer with a median failure time of 45 months [13]. However, a strict comparison with other reports on different treatment modalities is difficult because of the limitation and heterogeneity of each study. The present study has shown that biochemical failure occurred exclusively in cases with distant metastasis. This observation should be confirmed through a longer follow-up because local failure could occur later [14,15].

Biochemical failure and clinical failure were observed independent of the number of high-risk features. As Stone et al. suggested, the optimal BED for high-grade prostate cancer with a Gleason score of $8-10$ is 220 Gy by multicenter 
analysis [7]. The group also demonstrated that in a subset of biopsy-proven T3a, T3b, or N1 disease, the 7 year BFFS is $60 \%$ and $74 \%$ for BED below 200 Gy, and 200 Gy or above, respectively [10]. The present data has shown by a single institutional study that patients with high-risk and very highrisk prostate cancer, including N1 disease, show excellent biochemical control by receiving BED $>220 \mathrm{~Gy}$.

Toxicity in the present study is minimal when compared with the previous reports using $\operatorname{LDR}[3,11]$ and HDR $[12,13,14]$ in combination with EBRT. Although the total number of N1 disease patients in the present study was limited, the good biochemical control obtained using LDR in combination with whole pelvis EBRT is encouraging. Small nodal metastases may be well controlled with 45 Gy EBRT plus short-term ADT if the local prostate receives a high radiation dose (BED > 220 Gy), although much longer follow-up is required.

Our study limitations included: 1) short period of follow-up; 2) retrospective character of this study; 3) seminal vesicle and nodal involvement was diagnosed not by biopsy-proven, but by radiologic findings only. Even considering the above-mentioned shortcomings, this study has suggested that high-dose (BED > 220 Gy) radiotherapy by LDR in combination with EBRT may have an impact on BFFS in high-risk and very high-risk cancer.

Although a longer follow-up is necessary to validate the present findings, the reproducibility of this approach should be verified in order to use it as one of the optimal treatment modalities for high-risk and very high-risk prostate cancer patients.

\section{Conclusions}

High-dose (BED > 220 Gy) radiotherapy by LDR in combination with EBRT has shown an excellent outcome on BFFS in high-risk and very high-risk cancer patients, although causal relationship between BED and BFFS remain to be elucidated further.

\section{Funding information}

This work was supported partly by Research Grant from Takeda Science Foundation and Research Grant for the Princess Takamatsu Cancer Research.

\section{Acknowledgements}

We thank Prof. Yoshitaka Murakami, Department of Medical Statistics, Toho University for his advice on Kaplan-Meier analysis.

\section{Disclosure}

Keisei Okamoto is associated with the Department of Brachytherapy for Prostate Cancer endowed by Nihon Medi-Physics Co., Ltd. Akinori Wada, and Naoaki Kohn have no competing interest.

\section{References}

1. Kao J, Cesaretti JA, Stone NN et al. Update on prostate brachytherapy: long-term outcomes and treatment-related morbidity. Curr Urol Rep 2011; 12: 237-242.
2. Juloori A, Shah C, Stephans K et al. Evolving Paradigm of Radiotherapy for High-Risk Prostate Cancer: Current Consensus and Continuing Controversies. Prostate Cancer 2016; 2016: 2420786.

3. Stone NN, Cesaretti JA, Rosenstein B et al. Do high radiation doses in locally advanced prostate cancer patients treated with 103Pd implant plus external beam irradiation cause increased urinary, rectal, and sexual morbidity? Brachytherapy 2010; 9: 114-118.

4. Stock RG, Stone NN, Cesaretti JA et al. Biologically effective dose values for prostate brachytherapy: effects on PSA failure and posttreatment biopsy results. Int J Radiat Oncol Biol Phys 2006; 64: 527-533.

5. Morris WJ, Tyldesley HH. ASCENDE-RT: A multicenter, randomized trial of dose-escalated external beam radiation therapy (EBRT-B) versus low-dose rate brachytherapy (LDR-B) for men with unfavorable-risk localized prostate cancer. J Clin Oncol 2015; 33 (Suppl 7): abstract 3.

6. Muralidhar V, Xiang M, Orio PF et al. Brachytherapy boost and cancer-specific mortality in favorable high-risk versus other high-risk prostate cancer. J Contemp Brachytherapy 2016; 8: 1-6.

7. Stone NN, Potters L, Davis BJ et al. Multicenter analysis of effect of high biologic effective dose on biochemical failure and survival outcomes in patients with Gleason score 7-10 prostate cancer treated with permanent prostate brachytherapy. Int J Radiat Oncol Biol Phys 2009; 73: 341-346.

8. Stock RG, Stone NN, Wesson MF et al. A modified technique allowing interactive ultrasound-guided three-dimensional transperineal prostate implantation. Int J Radiat Oncol Biol Phys 1995; 32: 219-225.

9. Ho AY, Burri RJ, Jennings GT et al. Is seminal vesicle implantation with permanent sources possible? A dose-volume histogram analysis in patients undergoing combined 103Pd implantation and external beam radiation for $\mathrm{T} 3 \mathrm{c}$ prostate cancer. Brachytherapy 2007; 6: 38-43.

10. Carpenter TJ, Forsythe K, Kao J et al. Outcomes for patients with extraprostatic prostate cancer treated with trimodality therapy, including brachytherapy, external beam radiotherapy, and hormone therapy. Brachytherapy 2011; 10: 261-268.

11. Ohashi T, Yorozu A, Saito S et al. Combined brachytherapy and external beam radiotherapy without adjuvant androgen deprivation therapy for high-risk prostate cancer. Radiat Oncol 2014; 9: 13.

12. Martínez-Monge R, Moreno M, Ciérvide R et al. External-beam radiation therapy and high-dose rate brachytherapy combined with long-term androgen deprivation therapy in high and very high prostate cancer: preliminary data on clinical outcome. Int J Radiat Oncol Biol Phys 2012; 82: e469-476.

13. Kamrava M, Rwigema JC, Chung Met al. Predictors of distant metastasis after combined HDR brachytherapy and external beam radiation for prostate cancer. J Contemp Brachytherapy 2013; 5: 127-133.

14. Ishiyama H, Satoh T, Kitano M et al. High-dose-rate brachytherapy and hypofractionated external beam radiotherapy combined with long-term hormonal therapy for high-risk and very high-risk prostate cancer: outcomes after 5-year follow-up. J Radiat Res 2014; 55: 509-517.

15. Okamoto K. Application and limitation of LDR brachytherapy for prostate cancer. Nippon Rinsho 2016; 74: 542-545. 